\title{
Transoral thyroidectomy with a next generation flexible robotic system: A feasibility study in a cadaveric model
}

\author{
Jason Y. K. Chan ${ }^{1}$, Yoon W. Koh ${ }^{2}$, Jeremy Richmon ${ }^{3}$, Jaewook Kim ${ }^{4}$, F. Christopher Holsinger ${ }^{5}$, \\ Lisa Orloff ${ }^{5}$, Angkoon Anuwong ${ }^{6}$
}

${ }^{1}$ Department of Otorhinolaryngology, Head and Neck Surgery, The Chinese University of Hong Kong, Hong Kong, China; ${ }^{2}$ Department of Otorhinolaryngology, Yonsei University College of Medicine, Seoul, South Korea; ${ }^{3}$ Department of Otolaryngology-Head and Neck Surgery, Massachusetts Eye and Ear Infirmary, Harvard Medical School, Boston, Massachusetts, USA; ${ }^{4}$ Department of Otorhinolaryngology Head and Neck Surgery, Soonchunhyang University Hospital, College of Medicine, Seoul, South Korea; ${ }^{5}$ Division of Head and Neck Surgery, Department of Otolaryngology, Stanford University, Palo Alto, California, USA; ${ }^{6}$ Department of Surgery, Police General Hospital, Faculty of Medicine, Siam University, Bangkok, Thailand

Contributions: (I) Conception and design: JY Chan, FC Holsinger, A Anuwong; (II) Administrative support: JY Chan, FC Holsinger; (III) Provision of study materials or patients: All authors; (IV) Collection and assembly of data: All authors; (V) Data analysis and interpretation: JY Chan; (VI) Manuscript writing: All authors; (VII) Final approval of manuscript: All authors.

Correspondence to: Jason Y. K. Chan, MBBS. Department of Otorhinolaryngology, Head and Neck Surgery, The Chinese University of Hong Kong, Hong Kong, China. Email: jasonchan@ent.cuhk.edu.hk; Angkoon Anuwong, MD. Department of Surgery, Police General Hospital, Faculty of Medicine, Siam University, 492/1, Rama I Road, Pathumwan, Bangkok 10330, Thailand. Email: noii167@hotmail.com.

Background: Transoral endoscopic thyroidectomy provides access via the oral vestibule and gas insufflation to provide reliable remote access surgery to perform a total thyroidectomy. The da Vinci SP (Intuitive Surgical Inc., Sunnyvale, CA, USA) is a next generation flexible single port system that offers unique advantages over previous robotic rigid systems. Here we sought to evaluate the feasibility of performing transoral thyroidectomy with this next generation flexible robotic system.

Methods: Cadaveric dissection with gas insufflation to test the feasibility of performing transoral thyroidectomy with the da Vinci SP.

Results: A $2 \mathrm{~cm}$ incision was made in the oral vestibule and the working space created with a $1 \mathrm{~cm}$ central port and two lateral $5 \mathrm{~mm}$ ports. Then an extra small wound protector (Applied Medical, Rancho Santa Margarita, CA) was placed through the central incision after closure of the $5 \mathrm{~mm}$ ports. The robotic system was then deployed through the wound protector while insufflation was maintained at $\sim 6 \mathrm{mmHg}$. Three instrument arms were deployed. A fenestrated bipolar was used to grasp the thyroid gland while Maryland bipolars and monopolar scissors were used to mobilize each hemi lobe of the thyroid. The recurrent laryngeal nerves were seen and preserved bilaterally. After completion of the surgery and removal of the wound protector the vestibular incision was measured to be $3 \mathrm{~cm}$. Further dissection to identify the mental nerves identified each nerve to be $>1 \mathrm{~cm}$ from the lateral extent of the central incision.

Conclusions: In summary, it is feasible to perform a total thyroidectomy with gas insufflation utilizing this next generation flexible robotic system. Further evaluation will be needed to validate the clinical applicability of this technique.

Keywords: Transoral thyroidectomy; thyroidectomy; remote access; robotic surgery; Transoral Robotic Surgery (TORS)

Submitted Jul 25, 2019. Accepted for publication Oct 08, 2019.

doi: 10.21037 /gs.2019.10.13

View this article at: http://dx.doi.org/10.21037/gs.2019.10.13 


\section{Introduction}

The first generation of the da Vinci robotic surgical system (Intuitive Surgical Inc., Sunnyvale, CA, USA) was FDA approved for use in T1-2 lesions of the oropharynx and used with low surgical morbidity and mortality. However, the original system had certain constraints including the rigid, straight orientation and size of instruments, the inability to use all four instruments at once and the challenge in docking the patient side cart $(1,2)$. To address these issues a novel, flexible, single-arm robotic surgical system (da Vinci SP Surgical system, Model SP999; Intuitive Surgical Inc.) was developed and first evaluated clinically in 2010 on 19 patients undergoing urological surgery in a prospective Innovation, Development, Exploration, Assessment, Long-term Study (IDEAL) phase 1 clinical trial (3). Subsequently the system has also been evaluated for Transoral Robotic Surgery (TORS) by our groups, with early results demonstrating that the system is safe and feasible for TORS (4).

Transoral Endoscopic thyroidectomy Vestibular Approach (TOETVA) is a natural orifice transluminal endoscopic surgery (NOTES) procedure that has been popularized recently for thyroidectomy, given the ability to be reproduced safely at multi-centers globally (5-7). Briefly, the technique uses three ports with one $10 \mathrm{~mm}$ port for a 30-degree laparoscope with two additional $5 \mathrm{~mm}$ ports for instruments combined with $\mathrm{CO}_{2}$ insufflation of the anterior neck subplatysmal space (7). Subsequently the use of the da Vinci robotic system with rigid arms and camera system has been described with the camera and two instruments placed through the vestibule that is supplemented by an axillary port. Here we describe the first pre-clinical study using this updated novel single arm flexible robot to perform transoral robotic vestibular approach to the thyroid using a gas insufflation technique.

\section{Methods}

IRB and written consent were not required for this cadaveric study. This is a technical note describing a new approach to performing TOETVA using a novel flexible robotic system with gas insufflation in a cadaver (Figure S1). The initial dissection was performed under endoscopic guidance as in TOETVA to raise the initial subplatysmal planes. Briefly, a $2 \mathrm{~cm}$ incision was made at the center of the oral vestibule through the mentalis muscle, then a plane was developed over the periosteum of the mentum using monopolar cautery and Kelly clamp forceps. A
$10 \mathrm{~mm}$ trocar was then inserted followed by a $10 \mathrm{~mm}$ 30-degree laparoscope facing downwards. $\mathrm{CO}_{2}$ was used for insufflation and maintained at a pressure of $6 \mathrm{mmHg}$. Two vertical cuts $5 \mathrm{~mm}$ in size medial to the canines were then made vertically to allow the placement of two $5 \mathrm{~mm}$ trocars where a Maryland dissector and hook cautery was used to create the working space deep to the platysma. The $5 \mathrm{~mm}$ port incisions were then closed to limit the gas leakage through these two ports and an extra small wound protector (Applied Medical, Rancho Santa Margarita, CA, USA) was placed through the vestibular incision. The da Vinci SP was then docked to the bedside and the single port cannula was then secured to the wound protector as shown in Figure 1 outside the oral cavity, allowing for the deployment of both the elbow and wrist joints for the instrument arms similar to that as used for transoral robotic surgery as previously described (4). The 3 instrument arms were then maneuvered though the center of the wound protector into the neck, then the camera was maneuvered into the neck. The three instruments used included the Maryland bipolar, fenestrated bipolar graspers and monopolar scissors.

With the instruments in place the strap muscles were divided by cutting the midline raphe and retracted laterally with the fenestrated graspers (Figure 2). The thyroid isthmus was exposed and dissected with the monopolar scissors. The upper pole was then elevated with the Maryland bipolars and the recurrent laryngeal nerve identified. The inferior thyroid vessels were identified and divided with the Maryland bipolars. The thyroid gland was then removed through the initial $20 \mathrm{~mm}$ incision in the vestibule. Bilateral Central neck dissections were also performed safely with the recurrent laryngeal nerves preserved. During the case there was minimal smoke and spray noted that required suctioning, if this does occur a suction catheter can easily be placed through one $5 \mathrm{~mm}$ port to aid clearance of the smoke.

\section{Results}

A cadaver with no previous neck surgery aged 68 years old with a height of 65 inches and a weight of $105 \mathrm{lbs}$ was used with the da Vinci SP as described above. The distance from the tip of the cannula of the da Vinci SP to the vestibule incision was $13.0 \mathrm{~cm}$. The distance from the mentum to the sternal notch with the neck extended was $13.0 \mathrm{~cm}$. During the procedure a total thyroidectomy was performed without difficulty and gas insufflation maintained at $6 \mathrm{mmHg}$ of pressure with high flow $\mathrm{CO}_{2}$ insufflation. Both recurrent 


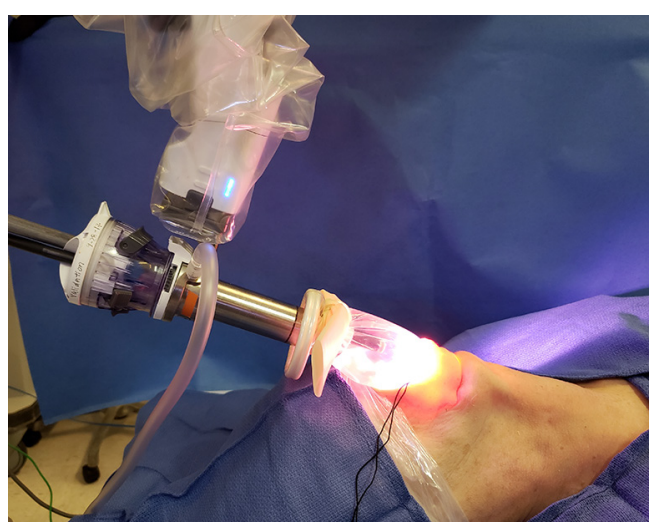

Figure 1 Docking of the single cannula through the wound protector with the camera and three arms deployed transorally to perform the thyroidectomy.

laryngeal nerves were identified and protected. At the end of the procedure the vestibular incision had widened to $3.5 \mathrm{~cm}$, but with further dissection the mental nerves were noted to still be far away from the initial vestibular incision.

\section{Discussion}

TOETVA has been described as a safe and feasible technique in performing remote access and cosmetically pleasing surgery to treat diseases of the thyroid glands (7). The advantage of this particular route over other remote access approaches to the thyroid gland include its central approach with the shortest distance to the thyroid gland, access to both lobes of the thyroid gland from the midline and no external visible scars. The application of robotics in transoral thyroid surgery offers several advantages over the endoscopic approach including camera control by the surgeon without the need for an assistant to constantly anticipate the surgeon's dissection (8). A high-definition, magnified immersive 3D view of the surgical field. Wristed instruments that allow for better angulation and orientation of instrumentations to maximize workspace. Finally, motion scaling and tremor filtration allow for the precise manipulation of tissues for dissection. In this regard, the transoral route has been used with the multiport da Vinci system to perform transoral surgery. However, there are still several drawbacks from using the current da Vinci Si and $\mathrm{Xi}$ robotic systems for transoral surgery given these were primarily designed to function in large work spaces as opposed to tight confined spaces. These include the instruments sizes being too large, the need for an extra

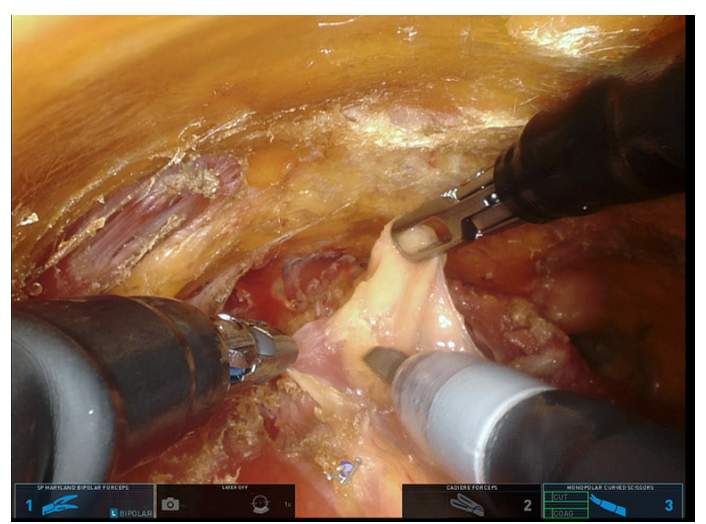

Figure 2 The camera and three instrument arms deployed in the neck transorally with gas insufflation to perform a thyroidectomy.

axillary port to facilitate the extra arm for retraction. The wider range of movement of the shaft laterally that leads to concerns of tearing of the lateral oral commissure and potentially temporary paraethesiae of the mental nerve.

Recently, with the advent of newer flexible robotic systems including the da Vinci single port system, these newer robotic platforms offer several potential advantages over the previous multiport systems. The single port cannula allows for the manipulation of three instruments and a camera through a $2.5 \mathrm{~cm}$ single cannula, that are flexible and of an appropriate smaller size with $6 \mathrm{~mm}$ instruments (1). This allows for a single incision in the oral vestibule centrally to deliver the tools needed for transoral robotic surgery. In this cadaver feasibility study, we have shown that gas insufflation utilizing the single port system is possible through an oral vestibule incision with the instruments deployed through the cannula $13.0 \mathrm{~cm}$ from the vestibular incision. Gas insufflation was maintained throughout the surgery without loss of pressure. With the 3 instrument arms deployed through the vestibular incision, the extra arm offered an advantage of retracting the strap muscles laterally without the need for an extra suture or axillary port that has been previously described $(7,8)$. The tennis ball sized workspace for the system is also ideal for space created deep to the platysma in performing the thyroidectomy.

Given the size of the $2.5 \mathrm{~cm}$ circular cannula, we used a wound protector to allow deployment of the instrument arms remotely as described for transoral robotic surgery, while maintaining gas insufflation $(1,4)$. Importantly, given the size of the cannula we noted that the incision widened 
from 2 to $3.5 \mathrm{~cm}$ but was still distant from the mental nerve foramen, with a further dissection following completion of the thyroidectomy and undocking of the robotic system. Thus, despite using this cannula it is likely that a permanent lower lip permanent paraesthesiae can be avoided but the potential for temporary hypesthesia of the lower lip is a potential that will need to be evaluated in further clinical studies $(5,7,8)$.

In summary in this cadaveric study it is feasible to use a next generation single port robotic system to perform a transoral robotic single port thyroidectomy through a vestibular approach using gas insufflation. Further study in a clinical setting will needed to be further evaluate the safety of using this system to perform transoral resections of the thyroid.

\section{Acknowledgments}

The authors acknowledge Jeff Smith, Michael Ikeda, Christie Draper, Melanie Yuan, Holly Liske, and the da Vinci SP Engineering Team.

\section{Footnote}

Conflicts of Interest: Dr's Chan, Koh, Richmon and Anuwong received travel support from Intuitive Surgical Inc. for the project. Dr. Kim, Orloff and Holsinger have no conflicts of interest or financial ties to disclose. The other authors have no conflicts of interest to declare.

Ethical Statement: The authors are accountable for all aspects of the work in ensuring that questions related

Cite this article as: Chan JYK, Koh YW, Richmon J, Kim J, Holsinger FC, Orloff L, Anuwong A. Transoral thyroidectomy with a next generation flexible robotic system: A feasibility study in a cadaveric model. Gland Surg 2019;8(6):644-647. doi: 10.21037 /gs.2019.10.13 to the accuracy or integrity of any part of the work are appropriately investigated and resolved.

\section{References}

1. Holsinger FC. A flexible, single-arm robotic surgical system for transoral resection of the tonsil and lateral pharyngeal wall: Next-generation robotic head and neck surgery. Laryngoscope 2016;126:864-9.

2. Chen MM, Orosco RK, Lim GC, et al. Improved transoral dissection of the tongue base with a next-generation robotic surgical system. Laryngoscope 2018;128:78-83.

3. Kaouk JH, Haber GP, Autorino R, et al. A novel robotic system for single-port urologic surgery: First clinical investigation. Eur Urol 2014;66:1033-43.

4. Chan JYK, Wong EWY, Tsang RK, et al. Early results of a safety and feasibility clini-cal trial of a novel singleport flexible robot for transoral robotic surgery. Eur Arch Otorhinolaryngol 2017;274:3993-6.

5. Russell JO, Clark J, Noureldine SI, et al. Transoral thyroidectomy and parathyroidectomy - A North American series of robotic and endoscopic transoral approaches to the central neck. Oral Oncol 2017;71:75-80.

6. Dionigi G, Chai YJ, Tufano RP, et al. Transoral endoscopic thyroid-ectomy via a vestibular approach: Why and how? Endocrine 2018;59:275-9.

7. Anuwong A. Transoral endoscopic thyroidectomy vestibular approach: A series of the first 60 human cases. World J Surg 2016;40:491-7.

8. Richmon JD, Kim HY. Transoral robotic thyroidectomy (TORT): Procedures and out-comes. Gland Surg 2017;6:285-9. 


\section{Supplementary}

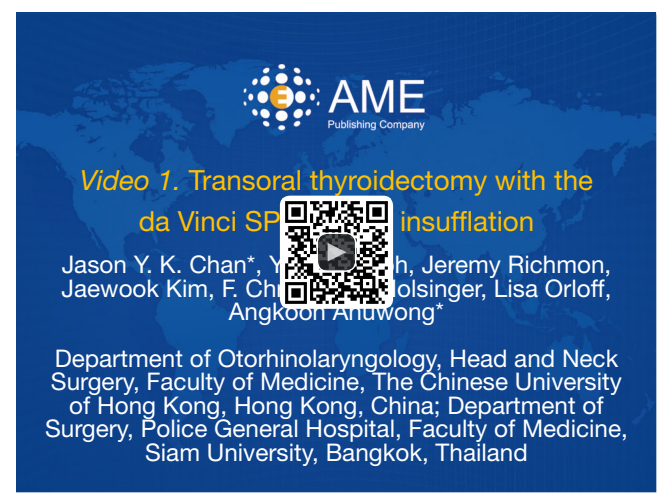

Figure S1 Transoral thyroidectomy with the da Vinci SP with gas insufflation (9).

Available online: http://www.asvide.com/watch/33032

\section{References}

9. Chan JY, Koh YW, Richmon J, et al. Transoral thyroidectomy with the da Vinci SP with gas insufflation. Asvide 2019;6:347. Available online: http://www.asvide.com/watch/33032 